\title{
Introduction to the intrinsic rolling with indefinite metric
}

\author{
Irina Markina AND FÁtima Silva Leite
}

\begin{abstract}
This paper concerns the rolling motion of one pseudo-Riemannian manifold over another. We compare the definition of rolling without slip and twist of two manifolds isometrically embedded into a pseudo-Euclidean space with the rolling defined only by intrinsic data, namely by metric tensors on manifolds. The smooth distribution on the configuration space, encoding the no-slip and no-twist kinematic conditions is constructed. Some results concerning the causal character of the rolling curves are also included. Several examples are presented along the paper to illustrate concepts and help to understand theoretical results.
\end{abstract}

\section{Introduction}

The study of nonholonomic systems composed of two n-dimensional manifolds rolling on each other, without slip and twist, pose many theoretical challenges and interesting control problems. In a Riemannian context, rolling has been approached from two viewpoints: either regarding the manifolds as subsets of an Euclidean space of higher dimension, or defining rolling intrinsically. The first viewpoint was developed in [13] followed by [9-11, 14]. An intrinsic formulation of rolling is the approach taken in [1-3, 6-8] for Riemannian manifolds.

Our paper generalizes from Riemannian to pseudo-Riemannian manifolds results that appeared in [8]. This required to check what remains valid and what needs to be fixed due to the appearance of new concepts and subtleties. Non-positiveness of the metric tensor, orientability issues, and causal

2000 Mathematics Subject Classification: 37J60, 53A17, 53A35.

Key words and phrases: Pseudo-Riemannian manifolds, pseudo-Euclidean space, rolling map, Christoffel symbols.

The first author was supported by NFR grants \#213440/BG and \#239033/F20. The second author was partially supported by ISR and grant PTDC/EEACRO/122812/2010. 
character of vectors are new ingredients that don't show up in the Riemannian case and force changes, first in the definition of rolling, and later in results throughout the paper. Several examples included in the paper show that the pseudo-Riemannian case can not be deduced from the Riemannian case simply by changing the name of the metric. While the indefiniteness of the metric provides new questions and challenges, this generalization also shows the strength of the presence of the metric tensor, independently of its sign definiteness.

The structure of the paper is the following. In Section 2 we review basic notations, present the definition of extrinsic rolling of manifolds isometrically embedded in $\mathbb{R}_{\nu}^{n}$, the pseudo-Euclidean space of dimension $n$ and index $\nu$, and study with some detail rolling motions of the Lorentzian sphere. We then proceed with the intrinsic definition of rolling in Section 3, where we compare the intrinsic component of the rolling map that depends only on metric data with the extrinsic part, that involves the information about an isometric embedding concretely chosen. In Section 4 we present the smooth distribution on the configuration space, which carries the kinematic restrictions of no-slip and no-twist, and study the causal character of the rolling map, in particular, giving some conditions under which the causal character of a rolling curve is preserved.

\section{Rolling submanifolds of pseudo-Euclidean spaces}

\subsection{Basic facts about pseudo-Riemannian geometry}

We review some basics about pseudo-Riemannian geometry and refer to O'Neill [12] for further details. Let $\mathbb{R}_{\nu}^{n}$ be the vector space $\mathbb{R}^{n}$ endowed with the pseudo-Euclidean metric induced by matrix $J=\operatorname{diag}\left(-I_{\nu}, I_{n-\nu}\right)$. That is, for any vectors $x, y \in \mathbb{R}^{n},\langle x, y\rangle_{J}=x^{\mathbf{t}} J y$, where $x^{\mathrm{t}}$ is $x$ transpose. We say that a vector $v \in \mathbb{R}_{\nu}^{n}$ is spacelike if $\langle v, v\rangle_{J}>0$ or $v=0$; $v$ is timelike if $\langle v, v\rangle_{J}<0$; $v$ is null if $\langle v, v\rangle_{J}=0$. The norm $|v|$ is defined to be $|v|:=$ $\left|\langle v, v\rangle_{J}\right|^{1 / 2}$.

Let $(M, g)$ be a submanifold of $\left(\mathbb{R}_{\nu}^{n},\langle., .\rangle_{J}\right)$ and $\imath: M \hookrightarrow \mathbb{R}_{\nu}^{n}$ the isometric embedding, see [4]. The tangent space $T_{x} M$ and the orthogonal complement $T_{x}^{\perp} M$ are nondegenerate spaces and

$$
\mathbb{R}_{\nu}^{n}=T_{x} M \oplus T_{x}^{\perp} M, \quad \forall x \in M
$$


The Levi-Civita connection $\bar{\nabla}$ on $\mathbb{R}_{\nu}^{n}$ induces the Levi-Civita connection $\nabla$ and the normal connection $\nabla^{\perp}$ on $M$ according to the following rules:

$$
\bar{\nabla}_{\bar{X}} \bar{Z}=\nabla_{X} Z+\left(\bar{\nabla}_{\bar{X}} \bar{Z}\right)^{\perp}, \quad \bar{\nabla}_{\bar{X}} \bar{\Psi}=\left(\bar{\nabla}_{\bar{X}} \bar{\Psi}\right)^{\top}+\nabla_{X}^{\perp} \Psi,
$$

for vector fields $X, Z, \Psi$ on $M$ and their extensions $\bar{X}, \bar{Z}, \bar{\Psi}$ on $\mathbb{R}_{\nu}^{n}$.

If $\gamma$ is an absolutely continuous curve in $M, Z$ is a smooth vector field tangent to $M$ along $\gamma$, and $\Psi$ is a smooth vector field normal to $M$ along $\gamma$, then the formulas (2) have their analogous in terms of covariant derivatives along $\gamma$ :

$$
\frac{\bar{D}}{d t} \bar{Z}=\frac{D}{d t} Z+\left(\frac{\bar{D}}{d t} \bar{Z}\right)^{\perp}, \quad \frac{\bar{D}}{d t} \bar{\Psi}=\left(\frac{\bar{D}}{d t} \bar{\Psi}\right)^{\top}+\frac{D^{\perp}}{d t} \Psi,
$$

where $\bar{Z}, \bar{\Psi}$ are extensions of $Z$ and $\Psi$ in a neighborhood of $\gamma$.

The covariant derivative $\frac{D}{d t}$ along an absolutely continuous curve $\gamma$ : $[a, b] \rightarrow M$ defines a tangent parallel vector field. For any initial value $Z(\gamma(a)) \in T_{\gamma(a)} M$ corresponds a unique tangent parallel vector field. It defines the linear isometry sending $Z(\gamma(a))$ to $Z(\gamma(b))$ that is called tangent parallel transport along $\gamma$. The analogous statement can be done for $\frac{D^{\perp}}{d t}$.

An absolutely continuous curve $t \mapsto \gamma(t)$ in $M$ is a geodesic if its velocity vector field is parallel along $\gamma$, i.e., $\nabla_{\dot{\gamma}} \dot{\gamma}(t)=0$ for almost all $t$. In pseudoRiemannian geometry there are three types of geodesics (spacelike, timelike or null), depending on the causal character of the initial velocity vector. The theory of pseudo-Riemannian geometry guarantees that a geodesic starting at $p_{0}$ with initial velocity $V_{0}$ is locally unique.

\subsection{Definition of rolling}

The present section is devoted to the geometric formulation of the rolling of a pseudo-Riemannian manifold $(M, g)$ over another $(\widehat{M}, \widehat{g})$, both connected, with the same dimension $m$ and index $\mu$ (the number of negative eigenvalues of $g$ and correspondingly of $\widehat{g}$ ). The manifolds are isometrically embedded in $\mathbb{R}_{\nu}^{n}$. A rolling motion of $M$ over $\widehat{M}$ is a rigid motion inside $\mathbb{R}_{\nu}^{n}$ and as such it is described by the action of the group $\overline{\mathbb{G}}=\mathbb{R}_{\nu}^{n} \rtimes \mathrm{O}_{\nu}(n)$ of the pseudo-Euclidean isometries of $\mathbb{R}_{\nu}^{n}\left[12\right.$, p. 240], where $\mathrm{O}_{\nu}(n)$ is the pseudoorthogonal group $\mathrm{O}_{\nu}(n)=\left\{X \in G L(n) \mid X^{\mathbf{t}} J X=J\right\}$. Elements in $\overline{\mathbb{G}}$ are pairs $(s, A)$, multiplication is defined as $\left(s_{1}, A_{1}\right)\left(s_{2}, A_{2}\right)=\left(s_{1}+A_{1} s_{2}, A_{1} A_{2}\right)$ and $(s, A)^{-1}=\left(-A^{-1} s, A^{-1}\right)$. The action of $\overline{\mathbb{G}}$ on $\mathbb{R}_{\nu}^{n}$ is defined by $(s, A) x=$ $s+A x$, for any $x \in \mathbb{R}_{\nu}^{n}$. 
Following the notations in [12] we define the $G$-orientation, where $G$ is one of the following subgroups of $\mathrm{O}_{\nu}(n)$ :

$$
\mathrm{O}_{\nu}^{++}(n) \cup \mathrm{O}_{\nu}^{--}(n), \quad \mathrm{O}_{\nu}^{++}(n) \cup \mathrm{O}_{\nu}^{+-}(n), \quad \mathrm{O}_{\nu}^{++}(n) \cup \mathrm{O}_{\nu}^{-+}(n)
$$

The connected component containing the identity is $\mathrm{O}_{\nu}^{++}(n)$ and it preserves time orientation, space orientation, and the orientation of the manifold. The Lie algebra $\mathfrak{o}_{\nu}(n)$ of $\mathrm{O}_{\nu}(n)$ is the set $\mathfrak{o}_{\nu}(n)=\left\{\mathcal{A} \in \mathfrak{g l}(n) \mid \mathcal{A}^{\mathbf{t}} J=-J \mathcal{A}\right\}$. We are now ready to generalize the classical definition of a rolling motion, as given in [8], which is an adaptation of the Euclidean definition in [13]. The following definition is indexed by the choice of one of the subgroups $G$ in (4), further denoted by $G_{\nu}(n)$. The Lie algebra of $G_{\nu}(n)$ is denoted by $\mathfrak{g}_{\nu}(n)$. The manifolds are assumed to be $G$-oriented.

Definition 1. A $G$-rolling of $M$ on $\widehat{M}$ without slip and twist is an absolutely continuous curve $(x, g):[0, \tau] \rightarrow M \times \mathbb{R}_{\nu}^{n} \rtimes G_{\nu}(n)$ satisfying the following conditions:

(i) $\widehat{x}(t):=g(t) x(t) \in \widehat{M}$ for almost every $t$,

(ii) $d_{x(t)} g(t) T_{x(t)} M=T_{\widehat{x}(t)} \widehat{M}$ for almost every $t$,

(iii) $\left.d_{x(t)} g(t)\right|_{T_{x(t)} M}: T_{x(t)} M \rightarrow T_{\widehat{x}(t)} \widehat{M}$ preserves $G$-orientation.

(iv) No slip condition: $\dot{\widehat{x}}(t)=d_{x(t)} g(t) \dot{x}(t)$, for almost every $t$.

(v) No twist condition (tangential part):

$$
d_{x(t)} g(t) \frac{D}{d t} Z(t)=\frac{D}{d t} d_{x(t)} g(t) Z(t),
$$

for any tangent vector field $Z(t)$ along $x(t)$ and almost every $t$.

(vi) No twist condition (normal part):

$$
d_{x(t)} g(t) \frac{D^{\perp}}{d t} \Psi(t)=\frac{D^{\perp}}{d t} d_{x(t)} g(t) \Psi(t),
$$

for any normal vector field $\Psi(t)$ along $x(t)$ and almost every $t$.

The curve $x$ is called the rolling curve, $\widehat{x}$ is called the development of $x$ on $\widehat{M}$. Due to the splitting (1), the condition (ii) implies that $d_{x(t)} g(t) T_{x(t)}^{\perp} M=$ $T_{\widehat{x}(t)}^{\perp} \widehat{M}$. 
The no twist conditions have an equivalent formulation (Proposition 1) involving the notion of parallel vector fields. The proof of this equivalence is straightforward.

Proposition 1. Assume that condition (ii) holds. Then, conditions (v) and (vi) are respectively equivalent to:

$\left(\mathrm{v}^{\prime}\right)$ A vector field $Z(t)$ is tangent parallel along $x(t)$ if and only if $d_{x(t)} g(t) Z(t)$ is tangent parallel along $\widehat{x}(t)$;

(vi') A vector field $\Psi(t)$ is normal parallel along $x(t)$ if and only if $d_{x(t)} g(t) \Psi(t)$ is normal parallel along $\widehat{x}(t)$.

We note that for manifolds of dimension one $\left(\mathrm{v}^{\prime}\right)$ is automatically satisfied, while for embeddings of codimension one (vi') always holds. From now on, whenever we write "rolling" we mean " $G$-rolling without slip and twist".

The following example of rolling an $m$-dimensional Lorentzian sphere on the affine tangent space at a point $x_{0}$, both embedded in the pseudoEuclidean space $\mathbb{R}_{1}^{m+1}$, is taken from [9].

\subsection{A benchmark example - the Lorentzian sphere $S_{1}^{m}$ rolling over the affine tangent space}

Let $M=S_{1}^{m}=\left\{x \in \mathbb{R}_{1}^{m+1}:\langle x, x\rangle_{J}=1\right\}$, and the affine tangent space $\widehat{M}=$ $T_{x_{0}}^{\text {aff }} S_{1}^{m}$. The following are easy consequence of definitions.

- $T_{x_{0}} S_{1}^{m}=\left\{v \in \mathbb{R}_{1}^{m+1}: v=\Omega x_{0}, \Omega \in \mathfrak{g}_{1}(m+1)\right\}$;

- $T_{x_{0}}^{\mathrm{aff}} S_{1}^{m}=\left\{v \in \mathbb{R}_{1}^{m+1}: v=x_{0}+\Omega x_{0}, \quad \Omega \in \mathfrak{g}_{1}(m+1)\right\}$;

- $T_{x_{0}}^{\perp} S_{1}^{m}=\operatorname{span}\left\{x_{0}\right\}$;

- $\operatorname{Ad}_{R}(\Omega)=R \Omega R^{-1} \in \mathfrak{g}_{1}(m+1)$, for every $R \in G_{1}(m+1)$ and $\Omega \in$ $\mathfrak{g}_{1}(m+1)$

- $\langle., .\rangle_{J}$ is $G_{1}(m+1)$-invariant.

- The Lie group $G_{1}(m+1)$ acts transitively on $S_{1}^{m}$, consequently any curve $t \mapsto x(t)$ satisfying $x(0)=x_{0}$ is of the form $x(t)=R(t) x_{0}$, for some $R(t) \in G_{1}(m+1)$ satisfying $R(0) x_{0}=x_{0}$. If, in particular, $R(0)=$ $I_{m+1}$, then $R(t)$ is a curve in $O_{1}^{++}(m+1)$.

2.3.1. Kinematic equations for rolling the Lorentzian sphere. Let $t \mapsto u(t)$ be an absolutely continuous function satisfying $\left\langle u(t), x_{0}\right\rangle_{J}=0$ and 
$t \mapsto(s(t), R(t)) \in \overline{\mathbb{G}}=\mathbb{R}_{1}^{m+1} \rtimes G_{1}(m+1)$ a curve in $\overline{\mathbb{G}}$, satisfying $(s(0), R(0))=$ $\left(0, I_{m+1}\right)$, with velocity vector given by

$$
\dot{s}(t)=u(t), \quad \dot{R}(t)=R(t)\left(u(t) x_{0}^{\mathrm{t}}-x_{0} u^{\mathrm{t}}(t)\right) J .
$$

We prove that $t \mapsto(x(t), g(t)) \in S_{1}^{m} \times \overline{\mathbb{G}}$, where $x(t)=R(t) x_{0}$ and $g(t)=$ $\left(s(t), R^{-1}(t)\right), R(t) \in O_{1}^{++}(m+1)$ is a rolling of $S_{1}^{m}$ over $T_{x_{0}}^{\text {aff }} S_{1}^{m}$, by showing that the first five conditions in Definition 1 hold. Equations (5) are called the kinematic equations for rolling the Lorentzian sphere over the affine tangent space at the point $x_{0}$. Condition (vi) is automatically satisfied since this is a co-dimension one case.

Proof of (i). We have $\widehat{x}(t):=g(t) x(t)=s(t)+R^{-1}(t) x(t)=s(t)+x_{0}$. Since $\dot{s}(t)=u(t) \in T_{x_{0}} S_{1}^{m}$ and $s(0)=0$, then $s(t) \in T_{x_{0}} S_{1}^{m}$ and $\widehat{x}(t)=s(t)+x_{0} \in$ $T_{x_{0}}^{\mathrm{aff}} S_{1}^{m}$.

Proof of (ii). Elements in $T_{x(t)} S_{1}^{m}$ are of the form $\Omega(t) x(t)$, with $\Omega(t) \in$ $\mathfrak{g}_{1}(m+1)$. So,

$$
d_{x(t)} g(t)(\Omega(t) x(t))=R^{-1}(t) \Omega(t) x(t)=\underbrace{R^{-1}(t) \Omega(t) R(t)}_{\in \mathfrak{g}_{1}(m+1)} x_{0} \in T_{x_{0}} S_{1}^{m} .
$$

Since $T_{\widehat{x}(t)}\left(T_{x_{0}}^{\text {aff }} S_{1}^{m}\right)$ is identified with $T_{x_{0}} S_{1}^{m}$, the result follows.

Proof of (iii). The map $d_{x(t)} g(t)=R^{-1}(t): T_{x(t)} S_{1}^{m} \rightarrow T_{\widehat{x}(t)} \widehat{M}$ is linear for all $t$ whenever it is defined. Since $R(t)$ is a continuous curve in $G_{1}(m+1)$ and $R(0)=I_{m+1}, R(t)$ and its inverse must remain in the connected component containing the identity of $G_{1}(m+1)$, which is $\mathrm{O}_{1}^{++}(m+1)$, so keeping the sign of the determinant for all $t$ that guarantees that $d_{x(t)} g(t)$ is orientation preserving.

Proof of (iv). We now have to use constraints on velocity given by (5).

$$
\begin{aligned}
d_{x(t)} g(t) \dot{x}(t) & =R^{-1}(t) \dot{x}(t)=R^{-1}(t) \dot{R}(t) x_{0}=\left(u(t) x_{0}^{\top}-x_{0} u^{\top}(t)\right) J x_{0} \\
& =\left\langle x_{0}, x_{0}\right\rangle_{J} u(t)-\left\langle u(t), x_{0}\right\rangle_{J} x_{0}=u(t) .
\end{aligned}
$$

On the other hand, $\widehat{x}(t)=s(t)+x_{0}$ from the proof of (i). So $\dot{\widehat{x}}(t)=\dot{s}(t)=$ $u(t)$, and the identity in (iv) holds.

Proof of (v). The covariant derivative of a tangent vector field $Z(t)$ along $x(t)$ is a tangent vector field along $x(t)$ that results from orthogonal projection of the extrinsic derivative $\dot{Z}(t)$ on the tangent space $T_{x(t)} S_{1}^{m}$. That is, 


$$
\begin{aligned}
& \frac{D}{d t} Z(t)=\dot{Z}(t)-\langle\dot{Z}(t), x(t)\rangle_{J} x(t) . \text { So, } \\
& \begin{aligned}
d_{x(t)} g(t) \frac{D}{d t} Z(t) & =R^{-1}(t)\left(\dot{Z}(t)-\langle\dot{Z}(t), x(t)\rangle_{J} x(t)\right) \\
& =R^{-1}(t) \dot{Z}(t)-\left\langle R^{-1}(t) \dot{Z}(t), R^{-1}(t) x(t)\right\rangle_{J} R^{-1}(t) x(t) \\
& =R^{-1}(t) \dot{Z}(t)-\left\langle R^{-1}(t) \dot{Z}(t), x_{0}\right\rangle_{J} x_{0} .
\end{aligned}
\end{aligned}
$$

On the other hand, since $d_{x(t)} g(t) Z(t)=R^{-1}(t) Z(t) \in T_{\widehat{x}(t)} \widehat{M} \cong T_{x_{0}} S_{1}^{m}$, we have

$$
\begin{aligned}
& \frac{D}{d t} d_{x(t)} g(t) Z(t)=\frac{D}{d t} R^{-1}(t) Z(t) \\
= & R^{-1}(t) Z(t)+R^{-1}(t) \dot{Z}(t)-\left\langle R^{-1}(t) Z(t)+R^{-1}(t) \dot{Z}(t), x_{0}\right\rangle_{J} x_{0} \\
= & R^{-1}(t) \dot{Z}(t)-\left\langle R^{-1}(t) \dot{Z}(t), x_{0}\right\rangle_{J} x_{0}+R^{-1}(t) Z(t)-\left\langle R^{-1}(t) Z(t), x_{0}\right\rangle_{J} x_{0} .
\end{aligned}
$$

So, in order to prove $(\mathrm{v})$ we have to show that the sum of the last two terms in the previous expression equals 0 . For this, take into consideration that $Z(t)=\Omega(t) x(t)=\Omega(t) R(t) x_{0}$, for some $\Omega(t) \in \mathfrak{g}_{1}(m+1)$, and $R^{-1}=$ $-R^{-1} \dot{R} R^{-1}$, to obtain

$$
\begin{aligned}
& R^{\cdot-1}(t) Z(t)=-R^{-1}(t)\left(u(t) x_{0}^{\mathbf{t}}-x_{0} u^{\mathbf{t}}(t)\right) J R^{-1}(t) \Omega(t) R(t) x_{0} \\
= & -\underbrace{\left\langle x_{0}, R^{-1}(t) \Omega(t) R(t) x_{0}\right\rangle_{J}}_{=0} u(t)+\left\langle u(t), R^{-1}(t) \Omega(t) R(t) x_{0}\right\rangle_{J} x_{0} \\
= & \left\langle u(t), R^{-1}(t) \Omega(t) R(t) x_{0}\right\rangle_{J} x_{0},
\end{aligned}
$$

and, consequently,

$$
\begin{aligned}
\left\langle R^{-1}(t) Z(t), x_{0}\right\rangle_{J} x_{0} & =\left\langle u(t), R^{-1}(t) \Omega(t) R(t) x_{0}\right\rangle_{J}\left\langle x_{0}, x_{0}\right\rangle_{J} x_{0} \\
& =\left\langle u(t), R^{-1}(t) \Omega(t) R(t) x_{0}\right\rangle_{J} x_{0}=R^{-1}(t) Z(t)
\end{aligned}
$$

completing the proof of $(\mathrm{v})$.

2.3.2. Rolling versus parallel transport. We show that parallel transport of a given vector $Y_{0}$ along a curve in $S_{1}^{m}$ can be realized by using the rolling along that curve. More precisely, we show that if $x(t)=R(t) x_{0}$ is a rolling curve satisfying the initial condition $x(0)=x_{0}$, rolling map $g(t)=$ $\left(s(t), R^{-1}(t)\right)$ with $g(0)=\left(0, I_{m+1}\right)$, and $Y_{0} \in T_{x_{0}} S_{1}^{m}$, then $Y(t)=R(t) Y_{0}$ is the unique tangent parallel vector field along $x(t)$ satisfying $Y(0)=Y_{0}$. Similarly, if $\Psi_{0} \in T_{x_{0}}^{\perp} S_{1}^{m}$, then $\Psi(t)=R(t) \Psi_{0}$ is the unique normal parallel vector field along the curve $x(t)$ satisfying $\Psi(0)=\Psi_{0}$. 
To prove the first statement, we notice that if $Y_{0} \in T_{x_{0}} S_{1}^{m}$, then $\langle Y(t), x(t)\rangle_{J}=\left\langle R(t) Y_{0}, R(t) x_{0}\right\rangle_{J}=\left\langle Y_{0}, x_{0}\right\rangle_{J}=0, \quad \Longrightarrow \quad Y(t) \in T_{x(t)} S_{1}^{m}$.

We now have to show that $\frac{D Y}{d t}=0$, where, in this case, $\frac{D Y(t)}{d t}=\dot{Y}(t)-$ $\langle\dot{Y}(t), x(t)\rangle_{J} x(t)$. Using the second kinematic equation in (5) and the conditions $\left\langle Y_{0}, x_{0}\right\rangle_{J}=0,\left\langle x_{0}, x_{0}\right\rangle_{J}=1$, we may conclude after simplifications that

$$
\begin{gathered}
\dot{Y}(t)=\dot{R}(t) Y_{0}=-\left\langle u(t), Y_{0}\right\rangle_{J} R(t) x_{0} \\
\text { and } \quad\langle\dot{Y}(t), x(t)\rangle_{J} x(t)=-\left\langle u(t), Y_{0}\right\rangle_{J} R(t) x_{0} .
\end{gathered}
$$

So, $\frac{D Y}{d t}=0$, i.e. $Y(t)=R(t) Y_{0}$ is the unique parallel vector field along $x(t)$ satisfying $Y(0)=Y_{0}$.

For the second statement, notice that if $\Psi_{0} \in T_{x_{0}}^{\perp} S_{1}^{m}$, then $\Psi_{0}=k x_{0}$, for some $k \in \mathbb{R}$, and consequently $\Psi(t)=R(t) \Psi_{0}=k x(t) \in T_{x(t)}^{\perp} S_{1}^{m}$. So, in this case, using similar arguments and the fact that $\left\langle u(t), x_{0}\right\rangle_{J}=0$, one has

$$
\langle\dot{\Psi}(t), x(t)\rangle_{J}=\left\langle\dot{R}(t) \Psi_{0}, x(t)\right\rangle_{J}=\left\langle\dot{R}(t) \Psi_{0}, R(t) x_{0}\right\rangle_{J}=k\left\langle u(t), x_{0}\right\rangle_{J}=0 .
$$

Consequently, $\frac{D^{\perp} \Psi}{d t}=\langle\dot{\Psi}(t), x(t)\rangle_{J} x(t)=0$, for almost all $t$, that is, $\Psi$ is the unique normal parallel vector field along $x(t)$ satisfying $\Psi(0)=\Psi_{0}$.

2.3.3. Causality. For the Lorentzian sphere, it can easily be shown that the rolling curve and its development have the same causal character. Indeed, using results from the previous subsection, namely $x(t)=R(t) x_{0}, \widehat{x}(t)=$ $s(t)+x_{0},\left\langle x_{0}, x_{0}\right\rangle_{J}=1,\left\langle u(t), x_{0}\right\rangle_{J}=0$, and the kinematic equations (5), we can write

$$
\begin{aligned}
\langle\dot{\widehat{x}}(t), \dot{\hat{x}}(t)\rangle_{J} & =\langle\dot{s}(t), \dot{s}(t)\rangle_{J}=\langle u(t), u(t)\rangle_{J} \\
\langle\dot{x}(t), \dot{x}(t)\rangle_{J} & =\left\langle\dot{R}(t) x_{0}, \dot{R}(t) x_{0}\right\rangle_{J} \\
& =\left\langle\left(u(t) x_{0}^{\mathrm{t}}-x_{0} u^{\mathrm{t}}(t)\right) J x_{0},\left(u(t) x_{0}^{\mathrm{t}}-x_{0} u^{\mathrm{t}}(t)\right) J x_{0}\right\rangle_{J} \\
& =\langle u(t), u(t)\rangle_{J} .
\end{aligned}
$$

Further, we want to show that the curve $t \in I \rightarrow R(t) \in \mathrm{O}_{1}(m+1)$ also has the same causal character, with respect to a scalar product in $\mathfrak{g l}(n)$ defined below. First, for any matrix $\mathcal{A} \in \mathfrak{g l}(n)$ and $J=\operatorname{diag}\left(-I_{\nu}, I_{n-\nu}\right)$ define the matrix $\mathcal{A}^{J}$ by

$$
\mathcal{A}^{J}:=J \mathcal{A}^{\mathrm{t}} J
$$

The Lie algebra $\mathfrak{g l}(n)$ may be equipped with a nondegenerate scalar product $\langle\langle\mathcal{A}, \mathcal{B}\rangle\rangle_{J}=\operatorname{tr}\left(A^{J} B\right)$ of signature $\nu$ that defines the causal structure on 
$\mathfrak{g l}(n)$. Notice that $\left(\mathcal{A}^{J}\right)^{J}=\mathcal{A}$, and $(\mathcal{A B})^{J}=\mathcal{B}^{J} \mathcal{A}^{J}$. Moreover, if $\mathcal{A} \in O_{\nu}(n)$, then $\mathcal{A}^{J} \mathcal{A}=\mathcal{A} \mathcal{A}^{J}=\mathrm{Id}$, which implies $\mathcal{A}^{J}=\mathcal{A}^{-1}$.

The Lie algebra $\mathfrak{o}_{\nu}(n)$ consists of $(n \times n)$ matrices satisfying $\mathcal{A}=-\mathcal{A}^{J}=$ $-J \mathcal{A}^{\mathbf{t}} J$. Consequently, one has $\mathcal{A}^{J} \mathcal{A}=\mathcal{A}^{J}=-\mathcal{A}^{2}$. The elements in $\mathfrak{o}_{\nu}(n)$ can be written as

$$
\mathcal{A}=\left(\begin{array}{cc}
a_{\nu} & b \\
b^{\mathbf{t}} & a_{n-\nu}
\end{array}\right), \quad a_{\nu} \in \mathfrak{o}(\nu), \quad a_{n-\nu} \in \mathfrak{o}(n-\nu) .
$$

So,

$$
\langle\langle\mathcal{A}, \mathcal{A}\rangle\rangle_{J}=\operatorname{tr}\left(\mathcal{A}^{J} \mathcal{A}\right)=-\operatorname{tr}\left(\mathcal{A}^{2}\right)=\operatorname{tr}\left(\begin{array}{cc}
-a_{\nu}^{2} & 0 \\
0 & -a_{n-\nu}^{2}
\end{array}\right)-2 \operatorname{tr}\left(b b^{\mathbf{t}}\right) .
$$

The first term involving the skew symmetric matrices $a_{\nu}$ and $a_{n-\nu}$ is always positive and represents the spacelike part. The matrix $b$ is responsible for the timelike character of elements of the Lie algebra $\mathfrak{o}_{\nu}(n)$.

We transfer this causal structure to the curves on the group $\mathrm{O}_{\nu}(n)$. Let $A: I \rightarrow \mathrm{O}_{\nu}(n)$ be a smooth curve. We say that the curve $A$ is spacelike, timelike or null if the product $\langle\langle\dot{A}, \dot{A}\rangle\rangle_{J}$ is positive, negative or equals zero, respectively. It can easily be checked that the scalar product $\langle\langle., .\rangle\rangle_{J}$ is $\mathrm{O}_{\nu}(n)$-invariant.

So, using the same ingredients as before and the kinematic equations (5), we conclude that for the rolling of $S_{1}^{m}$ on the affine tangent space one get

$$
\langle\langle\dot{R}(t), \dot{R}(t)\rangle\rangle_{J}=2\langle u(t), u(t)\rangle_{J}
$$

2.3.4. Controllability. The vector function $u(t)$ in the kinematic equations (5) is a control function. The choice of the controls defines the rolling curve. It has been proved in [9] that the kinematic equations (5) are completely controllable in $\mathbb{R}_{\nu}^{m+1} \times \mathrm{O}_{\nu}^{++}(m+1)$. More general results about sufficient conditions that guarantee the controllability of the rolling process can be found in $[3,5]$.

However, nothing guarantees that the causal character of the velocity vector remains invariant. The presence of the causal structure arises the natural problem to describe the set $\mathcal{R}_{x_{0}} \subset M$ of points reachable by a timelike (spacelike or null) curve from a given point $x_{0} \in M$. By this we mean that the sign of $\langle\dot{x}(t), \dot{x}(t)\rangle_{J}$ remains negative (positive or zero), for those $t>0$ where the velocity vector is defined. Before trying to answer this question we analyze a slightly different but simpler issue, that of geodesic reachability by rolling. 
Definition 2. We say that a point $x_{1} \in M$ is geodesically reachable by rolling from a point $x_{0} \in M$, if there exists a geodesic $x(t)=R(t) x_{0}$, with $R(t)$ a solution of the second kinematic equation in (5), satisfying $x(0)=x_{0}$, $x\left(t_{1}\right)=x_{1}$, for some $t_{1}>0$.

Since geodesics preserve their causal character it is easier to describe the subset of $\mathcal{R}_{x_{0}}$ reachable by geodesics. For the Lorentzian sphere, we characterize the set of points that can be geodesically reachable from a generic point $x_{0}$. First, we recall from [11] what are the geodesics in $S_{1}^{m}$ generated by $\mathcal{A}=\left(u x_{0}^{\mathrm{t}}-x_{0} u^{\mathbf{t}}\right) J$ with constant $u$ from the kinematic equations (5).

- If $\langle u, u\rangle_{J}=1$, then $x(t)=\exp (\mathcal{A} t) x_{0}=x_{0} \cos (t)+u \sin (t)$ is a spacelike geodesic satisfying $x(0)=x_{0}$.

- If $\langle u, u\rangle_{J}=-1$, then $x(t)=\exp (\mathcal{A} t) x_{0}=x_{0} \cosh (t)+u \sinh (t)$ is a timelike geodesic satisfying $x(0)=x_{0}$.

- If $\langle u, u\rangle_{J}=0$, then $x(t)=\exp (\mathcal{A} t) x_{0}=x_{0}+u t$ is a null geodesic satisfying $x(0)=x_{0}$.

Proposition 2. Let $x_{0}$ be any point in $S_{1}^{m}$. If $x_{1} \in S_{1}^{m}$ belongs to the set

$$
\left\{x \in S_{1}^{m} \text {, such that }\left\langle x_{0}, x\right\rangle_{J}>-1\right\} \cup\left\{-x_{0}\right\} \text {, }
$$

then $x_{1}$ is geodesically reachable from $x_{0}$.

Proof. The proof is constructive, in the sense that we construct the geodesic that realizes the job, according to the value of $\left\langle x_{0}, x_{1}\right\rangle_{J}$.

1) If $\left\langle x_{0}, x_{1}\right\rangle_{J}>1$, i.e., $\left\langle x_{0}, x_{1}\right\rangle_{J}=\cosh \theta$, for some $\theta \neq 0$, the timelike geodesic $x(t)=x_{0} \cosh (t)+u \sinh (t)$, where $u=\frac{x_{1}-x_{0} \cosh \theta}{\sinh \theta}$, links $x_{0}$ (at $t=0)$ to $x_{1}($ at $t=\theta)$. It is a simple calculation to show that, in this case, $\langle u, u\rangle_{J}=-1$ and we conclude that such kind of points are timelike reachable by geodesics.

$2)$ If $\left\langle x_{0}, x_{1}\right\rangle_{J}=1$, the null geodesic $x(t)=x_{0}+t u$, with $u=x_{1}-x_{0}$, links $x_{0}($ at $t=0)$ to $x_{1}($ at $t=1)$. In this case $\langle u, u\rangle_{J}=0$. Here we have example when $x_{1}$ is reachable by null geodesics.

3) If $\left.\left\langle x_{0}, x_{1}\right\rangle_{J} \in\right]-1,1\left[\right.$, i.e., $\left\langle x_{0}, x_{1}\right\rangle_{J}=\cos \theta$, for some $\theta \neq k \pi$, the spacelike geodesic $x(t)=x_{0} \cos (t)+u \sin (t)$, where $u=\frac{x_{1}-x_{0} \cos \theta}{\sin \theta}$, links $x_{0}$ (at $t=0)$ to $x_{1}($ at $t=\theta)$. In this case, $\langle u, u\rangle_{J}=1$. 
4) If $x_{1}=-x_{0}$, any spacelike geodesic $x(t)=x_{0} \cos (t)+u \sin (t)$, with $u$ satisfying $\left\langle x_{0}, u\right\rangle_{J}=0$, links $x_{0}($ at $t=0)$ to $x_{1}=-x_{0}($ at $t=\pi)$. The last two cases show the reachability by spacelike geodesics.

Remark 1. We can introduce the time orientation on $S_{1}^{m}$ by choosing a globally defined timelike vector field $T$. Then a timelike geodesic starting at $x_{0}$ and having property $\langle\dot{x}(0), T\rangle_{J}<0$ is called future directed and we introduce the notion of rolling along geodesic to the future. Moreover, when $\left\langle x_{0}, x_{1}\right\rangle_{J} \leq-1$ and $x_{1} \neq-x_{0}$, it is possible to reach $x_{1}$ from $x_{0}$ by a broken geodesic which change its causal character. For instance, first join $x_{0}$ to $-x_{1}$ by a timelike geodesic (if $\left\langle x_{0}, x_{1}\right\rangle_{J}>1$ ) or lightlike geodesic (if $\left\langle x_{0}, x_{1}\right\rangle_{J}=$ 1 ), and then join $-x_{1}$ to $x_{1}$ by a spacelike geodesic.

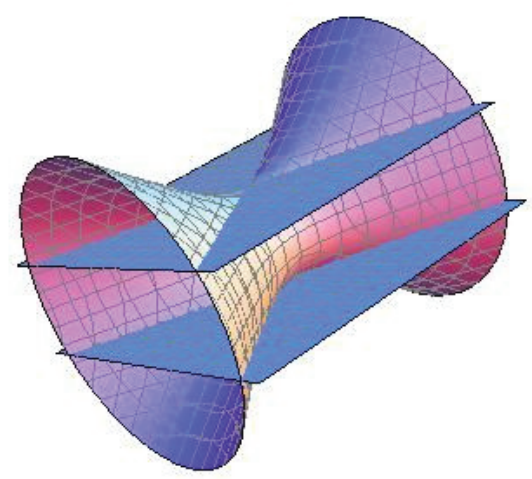

Figure 1: Partition of $S_{1}^{2}$ into causal/no-causal subsets from $x_{0}=(0,0,1)$.

Figure 1 shows the points that can be reached in this way from the point $x_{0}=(0,0,1) \in S_{1}^{2}$. Only $-x_{0}$ and points above the affine tangent space at $-x_{0}$ can be reached.

Based in the previous result, it is possible to give a precise geometric description of the reachable set from a point $x_{0}$, using spacelike geodesics only, and, similarly, timelike or null geodesics only. Two parallel hyperplanes in $\mathbb{R}_{1}^{m+1}$, make the correct separation, as the following shows.

Proposition 3. Let $x_{0}$ and $x_{1}$ be distinct arbitrary points in $S_{1}^{m}$. Then,

(1) $x_{1}$ is reachable from $x_{0}$ by a lightlike geodesic if and only if $x_{1} \in$ $T_{x_{0}}^{\mathrm{aff}} S_{1}^{m}$. 
(2) $x_{1}$ is reachable from $x_{0}$ by a timelike geodesic if and only if $x_{1}$ is on one side of the hyperplane $T_{x_{0}}^{\mathrm{aff}} S_{1}^{m}$, the side that doesn't contain $0 \in \mathbb{R}_{1}^{m+1}$.

(3) $x_{1}$ is reachable from $x_{0}$ by a spacelike geodesic if and only if $x_{1}$ lies between the hyperplanes $T_{x_{0}}^{\text {aff }} S_{1}^{m}$ and $T_{-x_{0}}^{\text {aff }} S_{1}^{m}$ or $x_{1}=-x_{0}$.

The proof is based on some simple facts.

The two images in Figure 2 indicate that the region accessible by spacelike geodesics narrows as the point $x_{0}$ moves away from the origin. For points at infinity, the two hyperplanes coincide and only timelike and null geodesics exist. We emphasize that the results obtained for the Lorentzian sphere can
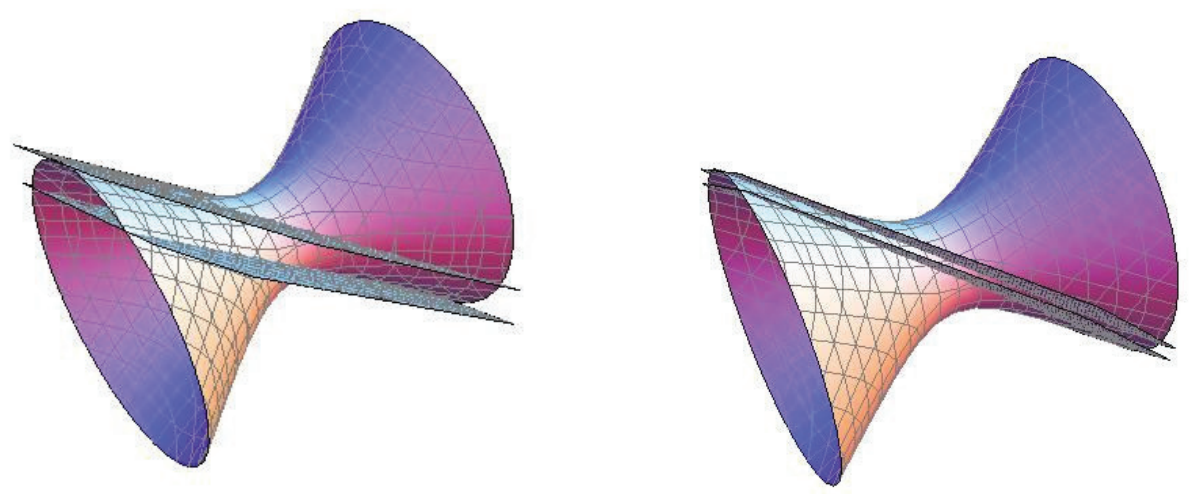

Figure 2: On the left $x_{0}=(2,2,1)$, on the right $x_{0}=(\sqrt{40}, 4,5)$.

be extended to other hyperquadrics.

\section{Intrinsic rolling}

\subsection{Reformulation of rolling in terms of bundles}

Let $W$ and $\widehat{W}$ be two oriented scalar product spaces with the same index $\mu$ and dimension $m$. We denote by $G(W, \widehat{W})$ any of the groups (4) of linear isometries between $W$ and $\widehat{W}$. When $W=\widehat{W}$, we write $G(W)$. For any pair $M$ and $\widehat{M}$ of $m$-dimensional connected oriented manifolds of the index $\mu$ we 
introduce the tangential part $Q$ of the configuration space

$$
Q=\left\{q \in G\left(T_{x} M, T_{\widehat{x}} \widehat{M}\right) \mid x \in M, \widehat{x} \in \widehat{M}\right\} .
$$

The space $Q$ is a fiber bundle over $M \times \widehat{M}$ of the dimension $2 m+(m(m-$ 1) $/ 2=\frac{m(m+3)}{2}$, where the group $G_{\mu}(m)$ acts on the fibers. Let $\iota: M \rightarrow \mathbb{R}_{\nu}^{n}=$ $\mathbb{R}_{\nu}^{m+\mathbf{m}}$ and $\widehat{\iota}: \widehat{M} \rightarrow \mathbb{R}_{\nu}^{m+\mathbf{m}}$ be two isometric embeddings. The normal part of the configuration space is the fiber bundle

$$
P_{\iota, \widehat{\iota}}:=\left\{p \in G\left(T_{x}^{\perp} M, T_{\widehat{x}}^{\perp} \widehat{M}\right) \mid x \in M, \widehat{x} \in \widehat{M}\right\} .
$$

of dimension $2 m+\frac{\mathbf{m}(\mathbf{m}-1)}{2}$ and the action of $G_{\nu-\mu}(\mathbf{m})$ on the fibers. While $Q$ is invariant of embeddings, the part $P_{\iota, \widehat{\iota}}$ depends on $\iota$ and $\widehat{\iota}$. By $Q \oplus P_{\iota, \widehat{\iota}}$ we denote the Whitney sum of the constructed above fiber bundles. By making use of the introduced fiber bundles we reformulate the Definition 1.

Definition 3. An extrinsic rolling (without slip and twist) of $M$ on $\widehat{M}$ along $x(t)$ and $\widehat{x}(t)$ is an absolutely continuous curve $(q, p):[0, \tau] \rightarrow Q \oplus P_{\iota, \widehat{\iota}}$ such that

(I) no slip condition: $\dot{\widehat{x}}(t)=q(t) \dot{x}(t)$ for almost every $t$,

(II) no twist condition (tangential part) $: q(t) \frac{D}{d t} Z(t)=\frac{D}{d t} q(t) Z(t)$ for any tangent vector field $Z(t)$ along $x(t)$ and almost every $t$,

(III) no twist condition (normal part) $: p(t) \frac{D^{\perp}}{d t} \Psi(t)=\frac{D^{\perp}}{d t} p(t) \Psi(t)$ for any normal vector field $\Psi(t)$ along $x(t)$ and almost every $t$.

Definitions 1 and 3 are equivalent due to the following proposition, whose proof literally follows the analogous statement in [8].

Proposition 4. If a curve $(x, g):[0, \tau] \rightarrow M \times \mathbb{R}_{\nu}^{m+\mathbf{m}} \rtimes G_{\nu}(m+\mathbf{m})$ satisfies the conditions (i)-(vi) in Definition 1, then the mapping

$$
t \mapsto\left(\left.d_{x(t)} g(t)\right|_{T_{x(t)} M},\left.d_{x(t)} g(t)\right|_{T_{x(t)} M^{\perp}}\right)=:(q(t), p(t)),
$$

defines a curve in $Q \oplus P_{\iota, \widehat{\iota}}$ satisfying the conditions (I)-(III) of Definition 3.

Conversely, if $(q, p):[0, \tau] \rightarrow Q \oplus P_{\iota, \widehat{\iota}}$ is an absolutely continuous curve satisfying (I)-(III), then there exists a unique rolling

$$
(x, g):[0, \tau] \rightarrow M \times \mathbb{R}_{\nu}^{m+\mathbf{m}} \rtimes G_{\nu}(m+\mathbf{m}),
$$

such that $\left.d_{x(t)} g(t)\right|_{T_{x(t)} M}=q(t)$ and $\left.d_{x(t)} g(t)\right|_{T_{x(t)} M^{\perp}}=p(t)$. 
Definition 4. An intrinsic rolling (without slip and twist) of $M$ over $\widehat{M}$, along $x(t)$ and $\widehat{x}(t)$, is an absolutely continuous curve $q:[0, \tau] \rightarrow Q$, with projections $x(t)=\operatorname{pr}_{M} q(t)$ and $\widehat{x}(t)=\operatorname{pr}_{\widehat{M}} q(t)$, satisfying:

(I') no slip condition: $\dot{\widehat{x}}(t)=q(t) \dot{x}(t)$ for almost all $t$,

(II') no twist condition: $Z(t)$ is a parallel tangent vector field along $x(t)$, if and only if $q(t) Z(t)$ is parallel along $\widehat{x}(t)$ for almost all $t$.

\subsection{Intrinsic and extrinsic rollings along the same curves}

Let $x:[0, \tau] \rightarrow M$ and $\widehat{x}:[0, \tau] \rightarrow \widehat{M}$ be two fixed curves. We denote by $\left\{e_{j}(t)\right\}_{j=1}^{m}$ an orthonormal frame field of parallel tangent vector fields along $x(t)$ and by $\left\{\epsilon_{\lambda}(t)\right\}_{\lambda=1}^{\mathbf{m}}$ an orthonormal frame field of normal parallel vector fields along $x(t)$. Similarly, along $\widehat{x}(t)$, we define parallel frames $\left\{\hat{e}_{i}\right\}_{i=1}^{m}$ and $\left\{\hat{\epsilon}_{\kappa}\right\}_{\kappa=1}^{\mathbf{m}}$.

Lemma 1. A curve $(q(t), p(t))$ in $Q \oplus P_{\iota, \widehat{\iota}}$ in the fibers over $(x(t), \widehat{x}(t))$, satisfies (II) and (III) if and only if the matrices

$$
A(t)=\left\{a_{i j}(t)\right\}=\left\{\left\langle\hat{e}_{i}, q(t) e_{j}\right\rangle_{J}\right\}, \quad B(t)=\left\{b_{\kappa \lambda}(t)\right\}=\left\{\left\langle\hat{\epsilon}_{\kappa}(t), p(t) \epsilon_{\lambda}(t)\right\rangle_{J}\right\},
$$

in parallel frames are constant.

The proof of the lemma follows from $\dot{A}(t)=\dot{B}(t)=0$, details see in [8].

Assume that a pair of curves $(x, \widehat{x}):[0, \tau] \rightarrow M \times \widehat{M}$ is the projection of an intrinsic rolling map $q(t)$. The following uniqueness question can be asked: are there other intrinsic rollings along the same curve $(x, \widehat{x})$ ? To answer the question we define the vector spaces $V=\{v(t)$ is a tangent parallel v.f. $\left.\langle v(t), \dot{x}(t)\rangle_{J}=0\right\}$ and $\widehat{V}=\left\{\widehat{v}(t)\right.$ is a tangent parallel v.f $\left.\langle\widehat{v}(t), \dot{\widehat{x}}(t)\rangle_{J}=0\right\}$.

Theorem 1. Let $q:[0, \tau] \rightarrow Q$ be a given intrinsic rolling map without slip and twist that is projected to $(x(t), \widehat{x}(t))$. Then $\operatorname{dim} V=\operatorname{dim} \widehat{V}$. Moreover,

(a) the map $q$ is the unique intrinsic rolling of $M$ over $\widehat{M}$ along $x(t)$ and $\widehat{x}(t)$ if and only if $\operatorname{dim} V \leq 1$,

(b) if $\operatorname{dim} V \geq 2$, all the rolling maps along $x(t)$ and $\widehat{x}(t)$ differ from $q$ by an element in $G(\widehat{V})$.

Proof. Choose the frame of parallel vector fields $\left\{e_{i}\right\}_{i=1}^{n}$ along $x(t)$ and define the parallel frame $\left\{\hat{e}_{i}\right\}_{i=1}^{n}$ along $\widehat{x}(t)$ by $q(t) e_{i}=\hat{e}_{i}$. Assume that the 
first $k$ vector fields of each frame are orthogonal to curves $x(t)$ and $\widehat{x}(t)$, respectively. Notice that $e_{1}, \ldots, e_{k}$ is a basis for $V$, and $\hat{e}_{1}, \ldots, \hat{e}_{k}$ is a basis for $\widehat{V}$. By Lemma 1 the corresponding matrix $A=\left\{a_{i j}\right\}=\left\langle\hat{e}_{i}, q e_{j}\right\rangle_{J}$ is the diagonal matrix with \pm 1 on diagonal according to the causal character of the basic vectors.

Writing $\dot{\widehat{x}}=\sum_{i=1}^{n} \dot{\widehat{x}}_{i}(t) \hat{e}_{i}(t)$ and $\dot{x}=\sum_{i=1}^{n} \dot{x}_{i}(t) e_{i}(t)$, we get $\dot{\widehat{x}}_{i}(t)=\dot{x}_{i}(t)$ for $i=1, \ldots, n$ and $\widehat{x}_{i}(t)=\dot{x}_{i}(t)=0$ for $i=1, \ldots, k$. So, if $\widetilde{q}$ is any other rolling, then $\widetilde{A}=\left\{\widetilde{a}_{i j}\right\}=\left\langle\hat{e}_{i}(t), \widetilde{q}(t) e_{j}(t)\right\rangle_{J}$ is clearly of the form

$$
\widetilde{A}=\left(\begin{array}{cc}
A^{\prime} & 0 \\
0 & J_{m-k}
\end{array}\right), \quad A^{\prime} \in G\left(\mathbb{R}_{\xi}^{k}\right),
$$

where $J_{m-k}$ is the $((m-k) \times(m-k))$ matrix with entires \pm 1 on the diagonal and 0 otherwise. The matrix $A^{\prime}$ is unique if $k$ is 0 or 1 . If $k \geq 2$, there is more freedom, since in the equality $\dot{\widehat{x}}_{i}=\sum_{j=1}^{n} \widetilde{a}_{i j} \dot{x}_{j}=\sum_{j=1}^{n} \widetilde{a}_{i j} \dot{\widehat{x}}_{j}$ the first $k$ values of $\dot{\hat{x}}_{j}$ vanish.

The converse also holds, that is, for any matrix $\widetilde{A}$ on the form (8), there is a rolling $\widetilde{q}$ corresponding to it.

Corollary 1. Assume that $x(t)$ is a geodesic in $M$. Then there exists an intrinsic rolling of $M$ on $\widehat{M}$ along $(x(t), \widehat{x}(t))$ if and only if $\widehat{x}(t)$ is a geodesic such that $\langle\dot{x}(t), \dot{x}(t)\rangle_{J}=\langle\dot{\widehat{x}}(t), \dot{\hat{x}}(t)\rangle_{J}$. Moreover, if $m \geq 2$, and if $\widehat{V}$ is defined as in Theorem 1, then

$$
\operatorname{dim} \widehat{V}=m-1
$$

and all the rollings along $x(t)$ and $\widehat{x}(t)$ differ by an element in $G(\widehat{V})$.

Proof. Calculating the covariant derivatives, and using the no-slip and notwist conditions (I')-(II'), we obtain

$$
\frac{D}{d t} \dot{\widehat{x}}(t)=\frac{D}{d t} q(t) \dot{x}(t)=q(t) \frac{D}{d t} \dot{x}(t) .
$$

Thus, the curve $x(t)$ is a geodesic if and only if $\widehat{x}(t)$ is also geodesic. The property (I') implies $\langle\dot{\widehat{x}}(t), \dot{\widehat{x}}(t)\rangle_{J}=\langle\dot{x}(t), \dot{x}(t)\rangle_{J}$. Conversely, equal speeds implies that $\dot{x}(t)$ differs from $\dot{\widehat{x}}(t)$ by an isometry $q(t): T_{x(t)} M \rightarrow T_{\widehat{x}}(t) \widehat{M}$ and the condition (I') follows.

Without loss of generality, we can suppose that $x$ is a timelike geodesic. We start the construction of rolling map by choosing the vector field $e_{m}(t)=$ $\frac{\dot{x}(t)}{\left|\langle\dot{x}(t) \dot{x}(t)\rangle_{J}\right|^{1 / 2}}$ that is parallel along $x(t)$. Pick the remaining $m-1$ parallel vector fields such that they form an orthonormal basis together with $e_{m}(t)$ 
along the curve $x(t)$. We repeat the same construction for a parallel frame $\left\{\hat{e}_{i}(t)\right\}_{i=1}^{m}$ along $\widehat{x}(t)$. Define the intrinsic rolling $q(t)$ by

$$
\begin{aligned}
& \left\langle\hat{e}_{m}(t), q(t) e_{j}(t)\right\rangle_{J}=\left\langle\hat{e}_{j}(t), q(t) e_{m}(t)\right\rangle_{J}=-\delta_{m, j}, \\
& A^{\prime}=\left\{\left\langle\hat{e}_{i}(t), q(t) e_{j}(t)\right\rangle_{J}\right\}_{i, j=1}^{m-1}
\end{aligned}
$$

where $A^{\prime} \in G_{\mu-1}(m-1)$ will be a constant matrix. Conversely, we can construct a rolling by formulas (9) starting from $A^{\prime} \in G_{\mu-1}(m-1)$.

Remark 2. We denote $E=\left\{\epsilon(t)\right.$ is a normal parallel v. f. $\langle\epsilon(t), \dot{x}(t)\rangle_{J}=$ $0\}$, and $\widehat{E}=\left\{\widehat{\epsilon}(t)\right.$ is a normal parallel v. f. $\left.\langle\widehat{\epsilon}(t), \widehat{\dot{x}}(t)\rangle_{J}=0\right\}$, where the scalar product is defined by the imbeddings. Both vector spaces have dimension $\mathbf{m}$. An extrinsic rolling $(q, p)$ extending an intrinsic rolling $q$ is determined up to a left action of $G(\widehat{E})$ or, equivalently, up to a right action of $G(E)$. Both $G(E)$ and $G(\widehat{E})$ are isomorphic to $G_{\nu-\mu}(\mathbf{m})$, but not canonically.

The following theorem concerns the uniquenes question of the extension of an intrinsic rolling to the extrinsic one.

Theorem 2. Let $q:[0, \tau] \rightarrow Q$ be an intrinsic rolling and let $\iota: M \rightarrow \mathbb{R}_{\nu}^{m+\mathbf{m}}$ and $\widehat{\iota}: \widehat{M} \rightarrow \mathbb{R}_{\nu}^{m+\mathbf{m}}$ be given isometric embeddings. Then, given an initial normal configuration

$$
p_{0} \in\left(P_{\iota, \widehat{\iota}}\right)_{\left(x_{0}, \widehat{x}_{0}\right)} \text {, where }\left(x_{0}, \widehat{x}_{0}\right)=\operatorname{pr}_{M \times \widehat{M}} q(0),
$$

there exists a unique extrinsic rolling $(q, p):[0, \tau] \rightarrow Q \oplus P_{\iota, \widehat{\iota}}$ satisfying $p(0)=p_{0}$.

Proof. Let $B_{0} \in G_{\nu-\mu}(\mathbf{m})$ be defined by $B_{0}=\left(b_{\kappa \lambda}\right)=\left(\left\langle\widehat{\epsilon}_{\kappa}(0), p_{0} \epsilon_{\lambda}(0)\right\rangle_{J}\right)$, with $\left\{\epsilon_{\lambda}(t)\right\}_{\lambda=1}^{\nu}$ and $\left\{\hat{\epsilon}_{\kappa}(t)\right\}_{\kappa=1}^{\nu}$ normal parallel frames along $x(t)$ and $\widehat{x}(t)$, respectively. Then $p(t)$ satisfies $b_{\kappa \lambda}=\left\langle\widehat{\epsilon}_{\kappa}(t), p(t) \epsilon_{\lambda}(t)\right\rangle_{J}$, by Lemma 1 , and it is uniquely determined by this.

\section{Distributions and causal character of the intrinsic rolling}

\subsection{Distributions}

The aim of this Section is to formulate the kinematic conditions for rolling without slip and without twist in terms of a distribution or subbundle of 
$T\left(Q \oplus P_{\iota, \widehat{\iota}}\right)$. Consider the local trivialization

$$
\begin{aligned}
Q \oplus P_{\iota, \widehat{\iota}} \supset \pi^{-1}(U \times \widehat{U}) & \stackrel{h}{\rightarrow} U \times \widehat{U} \times G_{\mu}(m) \times G_{\nu-\mu}(\mathbf{m}) \\
(q(t), p(t)) & \mapsto(x(t), \widehat{x}(t), A(t), B(t)),
\end{aligned}
$$

where $t$ belongs to rather small interval and

$$
A=\left(a_{i j}\right)_{i, j=1}^{m}=\left(\left\langle q e_{j}, \hat{e}_{i}\right\rangle_{J}\right)_{i, j=1}^{m}, \quad B=\left(b_{\kappa \lambda}\right)_{\kappa, \lambda=1}^{\mathbf{m}}=\left(\left\langle p \epsilon_{\lambda}, \hat{\epsilon}_{\kappa}\right\rangle_{J}\right)_{\kappa, \lambda=1}^{\mathbf{m}} .
$$

Here $\left\{e_{j}\right\}_{j=1}^{m},\left\{\epsilon_{\lambda}\right\}_{\lambda=1}^{\mathbf{m}},\left\{\hat{e}_{i}\right\}_{i=1}^{m}$ and $\left\{\hat{\epsilon}_{\kappa}\right\}_{\kappa=1}^{\mathbf{m}}$ are oriented orthonormal frames of vector bundles of $\left.T M\right|_{U},\left.T^{\perp} M\right|_{U},\left.T \widehat{M}\right|_{\widehat{U}}$ and $\left.T^{\perp} \widehat{M}\right|_{\widehat{U}}$, respectively. Moreover, we assume that the first $\mu$ terms of $\left\{e_{j}\right\}_{j=1}^{m}$ and $\left\{\hat{e}_{i}\right\}_{i=1}^{m}$ are timelike. Correspondingly, the first $\nu-\mu$ vector fields $\left\{\epsilon_{\lambda}\right\}_{\lambda=1}^{\mathbf{m}}$ and $\left\{\hat{\epsilon}_{\kappa}\right\}_{\kappa=1}^{\mathbf{m}}$ are also timelike.

The kinematic conditions (I)-(III) are written as restrictions on the velocity vector:

$$
\begin{aligned}
\dot{\gamma}(t) & =(\dot{x}(t), \dot{\widehat{x}}(t), \dot{A}(t), \dot{B}(t)) \\
& \in T_{x(t)} U \times T_{\widehat{x}(t)} \widehat{U} \times T_{A(t)} G_{\mu}(m) \times T_{B(t)} G_{\nu-\mu}(\mathbf{m}) .
\end{aligned}
$$

The Lie algebra $\mathfrak{g}_{\mu}(m)$ of the group $G_{\mu}(m)$ is spanned by

$$
\begin{aligned}
& \left\{W_{i j}=\frac{\partial}{\partial a_{i j}}-\varepsilon_{i} \varepsilon_{j} \frac{\partial}{\partial a_{j i}}, 1 \leq i<j \leq m\right\}, \\
& \varepsilon_{i}= \begin{cases}-1 & \text { if } \quad 1 \leq i \leq \mu, \\
1 & \text { if } \quad \mu+1 \leq i \leq m,\end{cases}
\end{aligned}
$$

The left invariant vector fields obtained by the transports of $W_{i j}(1)$ by $A \in$ $G_{\mu}(m)$ are the following

$$
A \cdot W_{i j}(1)=\sum_{r=1}^{m}\left(a_{r i} \frac{\partial}{\partial a_{r j}}-\varepsilon_{i} \varepsilon_{j} a_{r j} \frac{\partial}{\partial a_{r i}}\right)
$$

Consider the image of $\gamma(t)$, satisfying the conditions (I)-(III), under the trivialization.

$$
\dot{\gamma}(t)=\dot{x}(t)+\dot{\widehat{x}}(t)+\sum_{i, j=1}^{m} \dot{a}_{i j} \frac{\partial}{\partial a_{i j}}+\sum_{\kappa, \lambda=1}^{\mathbf{m}} \dot{b}_{\kappa \lambda} \frac{\partial}{\partial b_{\kappa \lambda}} .
$$

We want to write the last two terms in (13) in the left invariant bases of $T G_{\mu}(m)$ and $T G_{\nu-\mu}(\mathbf{m})$, based on conditions (II) and (III). Condition (II) 
holds if and only if $q \frac{D}{d t} e_{j}(x(t))=\frac{D}{d t} q e_{j}(x(t))$, which yields

$$
\sum_{i, j=1}^{m} \dot{a}_{i j} \frac{\partial}{\partial a_{i j}}=\sum_{i<j}\left(\left\langle\nabla_{\dot{x}(t)} e_{j}, e_{i}\right\rangle_{J}-\left\langle\nabla_{q \dot{x}(t)} q e_{j}, q e_{i}\right\rangle_{J}\right) \varepsilon_{i} A \cdot W_{i j} .
$$

Similarly, (III) holds if and only if

$$
\sum_{\kappa, \lambda=1}^{\mathbf{m}} \dot{b}_{\kappa \lambda} \frac{\partial}{\partial b_{\kappa \lambda}}=\sum_{\kappa<\lambda}\left(\left\langle\nabla_{\dot{x}(t)}^{\perp} \epsilon_{\lambda}, \epsilon_{\kappa}\right\rangle_{J}-\left\langle\nabla_{q \dot{x}(t)}^{\perp} p \epsilon_{\lambda}, p \epsilon_{\kappa}\right\rangle_{J}\right) \varepsilon_{\kappa} B \cdot W_{\kappa \lambda}
$$

We omit here technical details, that are very similar to those in [8].

Observe that, due to the expressions (14) and (15), the vector field $\dot{\gamma}$ along $\gamma$ on $Q \oplus P_{\iota, \widehat{\iota}}$ can be considered as a "non-twisted lift" of the vector field $\dot{x}$ along the curve $x(t)$ on $M$. We generalize this property on any local vector field on $M$.

Definition 5. Non-twisted lifts of a vector field $X$ on $U \subset M$ are the vector fields $\mathcal{V}(X)$ and $\mathcal{V}^{\perp}(X)$ on $\pi^{-1}(U \times \widehat{U}) \subset Q \oplus P_{\iota, \widehat{\iota}}$ satisfying

$$
\begin{aligned}
d h(\mathcal{V}(X)(q, p)) & =\sum_{i<j}\left(\left\langle\nabla_{X} e_{j}, e_{i}\right\rangle_{J}-\left\langle\nabla_{q X} q e_{j}, q e_{i}\right\rangle_{J}\right) \varepsilon_{i} A \cdot W_{i j} \\
d h\left(\mathcal{V}^{\perp}(X)(q, p)\right) & =\sum_{\kappa<\lambda}\left(\left\langle\nabla_{X}^{\perp} \epsilon_{\lambda}, \epsilon_{\kappa}\right\rangle_{J}-\left\langle\nabla_{q X}^{\perp} p \epsilon_{\lambda}, p \epsilon_{\kappa}\right\rangle_{J}\right) \varepsilon_{\kappa} B \cdot W_{\kappa \lambda} .
\end{aligned}
$$

for any local trivialization $h$ as in (10) and any $(q, p) \in \pi^{-1}(U \times \widehat{U})$.

Notice that since the covariant derivative along a vector field $X$ depends only on the value $X(x)$ at $x \in U \subset M$ we conclude that if $Y(x)=X(x)=$ $v_{x} \in T_{x} M$, then $\mathcal{V}(Y)(q, p)=\mathcal{V}(X)(q, p)$ for every $(q, p) \in\left(Q \oplus P_{\iota, \widehat{\iota}}\right)_{x \times \widehat{x}}$. Hence, we may define the lift $\mathcal{V}\left(v_{x}\right)(q, p)$ for any vector $v_{x} \in T_{x} M$ and $(q, p) \in\left(Q \oplus P_{\iota, \widehat{\iota}}\right)_{x \times \widehat{x}}$. The no-slip conditions imply that $q v_{x} \in T_{\widehat{x}} \widehat{M}$. Also notice that the map $X \mapsto \mathcal{V}(X)$ is linear. The same holds for $X \mapsto \mathcal{V}^{\perp}(X)$. This leads to the definition of the distributions contained in the following propositions.

Proposition 5. A curve $(q(t), p(t))$ in $Q \oplus P_{\iota, \widehat{\iota}}$ is a rolling if and only if it is a horizontal curve with respect to the distribution $E$, defined by

$$
\begin{aligned}
& E_{(q, p)}=\left\{v_{x}+q v_{x}+\mathcal{V}\left(v_{x}\right)(q, p)+\mathcal{V}^{\perp}\left(v_{x}\right)(q, p) \mid v_{x} \in T_{x} M\right\} \\
& (q, p) \in\left(Q \oplus P_{\iota, \widehat{\iota}}\right)_{x \times \widehat{x}}
\end{aligned}
$$


Proposition 6. A curve $q(t)$ in $Q$ is an intrinsic rolling if and only if it is a horizontal curve with respect to the distribution $D$, defined by

$$
D_{q}=\left\{v_{x}+q v_{x}+\mathcal{V}\left(v_{x}\right)(q) \mid v_{x} \in T_{x} M\right\}, \quad q \in Q_{x \times \widehat{x}}
$$

\subsection{Causal character of the rolling}

The specific feature of pseudo-Riemannian manifolds is the causal structure, or partition of all vectors into three classes: timelike, spacelike and nullike. It is easy to see the following

Proposition 7. If a rolling curve $x: I \rightarrow M$ is of one of the causal types, then the development curve $\hat{x}$ is of the same type.

Proof. Since the map $q: T_{x(t)} M \rightarrow T_{\hat{x}(t)} \widehat{M}$ is an isometry then the no-slip condition implies $\langle\dot{\hat{x}}(t), \dot{\hat{x}}(t)\rangle_{J}=\langle q(t) \dot{x}(t), q(t) \dot{x}(t)\rangle_{J}=\langle\dot{x}(t), \dot{x}(t)\rangle_{J}$.

The pseudo-orthogonal group also admits the scalar product as was mentioned in Subsection 2.3.3 that we denoted by $\langle\langle., .\rangle\rangle_{J}$. Under the local trivialization $h$ as in (10) a rolling curve takes the form $h(q(t), p(t))=$ $\gamma(t)=(x(t), \hat{x}(t)), A(t), B(t)), t \in I$. We know that the curves $x$ and $\hat{x}$ have the same causal character. We ask whether the curves $A \in G_{\mu}(m)$ and $B \in G_{\nu-\mu}(\mathbf{m})$ have the same causal character? As we saw the Lorentzian sphere and the hyperquadrics, in general, possess this property.

Theorem 3. If $\gamma(t)=(x(t), \hat{x}(t), A(t), B(t))$ is a rolling curve under the local trivialization then the causal character of curves $A(t), B(t)$ can be calculated as follows. The curve $A$ is timelike (spacelike or null) if the expression

$$
\sum_{i, h=1}^{m} \varepsilon_{i} \varepsilon_{h}\left(\sum_{k=1}^{m}\left[\dot{x}^{k} \Gamma_{k h}^{i}-c_{i h} \dot{\hat{x}^{k}} \widehat{\Gamma}_{k h}^{i}\right]\right)^{2},
$$

is negative (positive or zero), respectively. Here $c_{i h}=\sum_{r, s=1}^{m} \varepsilon_{r} \varepsilon_{s} a_{r h} a_{s i}$. The curve $B$ is timelike (spacelike or null) if the expression

$$
\sum_{\kappa, \chi=1}^{\mathbf{m}} \varepsilon_{\kappa} \varepsilon_{\chi}\left(\sum_{l=1}^{\mathbf{m}}\left[\dot{x}^{l}\left(\Gamma^{\perp}\right)_{l \chi}^{\kappa}-d_{\kappa \chi} \dot{\hat{x}}^{l}\left(\widehat{\Gamma}^{\perp}\right)_{l \chi}^{\kappa}\right]\right)^{2}
$$

is negative (positive or zero), respectively. Here $d_{\kappa \chi}=\sum_{\rho, \sigma=1}^{\mathbf{m}} \varepsilon_{\rho} \varepsilon_{\sigma} b_{\rho \chi} b_{\sigma \kappa}$. 
Proof. If $A: I \rightarrow G_{\mu}(m)$ then $\dot{A}(t)=A(t) \cdot U(t)$, where $U$ is a curve in the Lie algebra $\mathfrak{g}_{\mu}(m)$. Then $\langle\langle\dot{A}(t), \dot{A}(t)\rangle\rangle_{J}=\operatorname{tr}\left(\dot{A}^{J} \dot{A}\right)=-\operatorname{tr} U^{2}$, since $A^{\mathrm{t}} J A=$ $J$ and $J U^{\mathbf{t}} J=-U$. We start from study of $\dot{A}=\left\{\dot{a}_{i j}\right\}$ in (13). According to (14) we have $\dot{A}=A \cdot U$ with

$$
U=\sum_{i<j}\left(\left\langle\nabla_{\dot{x}(t)} e_{j}, e_{i}\right\rangle_{J}-\left\langle\nabla_{q \dot{x}(t)} q e_{j}, q e_{i}\right\rangle_{J}\right) \varepsilon_{i} W_{i j}(1)
$$

We denote the coefficients of $\varepsilon_{i} W_{i j}(1)$ by

$$
w_{i j}=\left(\left\langle\nabla_{\dot{x}(t)} e_{j}, e_{i}\right\rangle_{J}-\left\langle\nabla_{q \dot{x}(t)} q e_{j}, q e_{i}\right\rangle_{J}\right)
$$

Observe that $\left\langle\nabla_{\dot{x}(t)} e_{j}, e_{i}\right\rangle_{J}=\sum_{k=1}^{m} \dot{x}^{k}(t)\left\langle\nabla_{e_{k}} e_{j}, e_{i}\right\rangle_{J}=\sum_{k=1}^{m} \dot{x}^{k}(t) \Gamma_{k j}^{i}(x(t))$ and

$$
\begin{aligned}
\left\langle\nabla_{q \dot{x}(t)} q e_{j}, q e_{i}\right\rangle_{J} & =\sum_{l=1}^{m} \dot{\hat{x}}^{l}(t)\left\langle\nabla_{\widehat{e}_{l}} \sum_{r=1}^{m} \varepsilon_{r} a_{r j} \widehat{e}_{j}, \sum_{s=1}^{m} \varepsilon_{s} a_{s i} \widehat{e}_{i}\right\rangle_{J} \\
& =\sum_{s, r=1}^{m} \varepsilon_{r} \varepsilon_{s} a_{r j} a_{s i} \sum_{l=1}^{m} \dot{\hat{x}}^{l} \widehat{\Gamma}_{l j}^{i}(x(t)),
\end{aligned}
$$

where $\Gamma_{k j}^{i}(x(t))$ and $\widehat{\Gamma}_{l j}^{i}(x(t))$ are Christoffel symbols of Levi-Civita connections for $M$ and $\hat{M}$ along curves $x$ and $\widehat{x}$, respectively.

Since for the trace we need only information about the diagonal terms of $U^{2}$ we find

$$
\begin{aligned}
& \left\{U^{2}\right\}_{i i}=\sum_{h=1}^{m}\{U\}_{i h}\{U\}_{h i}=\sum_{h=1}^{m} \varepsilon_{h} w_{i h}^{2} \quad \text { for } \quad i=1, \ldots \mu . \\
& \left\{U^{2}\right\}_{i i}=\sum_{h=1}^{m}\{U\}_{i h}\{U\}_{h i}=\sum_{h=1}^{m}-\varepsilon_{h} w_{i h}^{2} \quad \text { for } \quad i=\mu, \ldots m .
\end{aligned}
$$

Thus the trace is expressed as followed

$$
-\operatorname{tr} U^{2}=-\sum_{i, h=1}^{m}-\varepsilon_{i} \varepsilon_{h} w_{i h}^{2}=\sum_{i, h=1}^{m} \varepsilon_{i} \varepsilon_{h}\left(\sum_{k=1}^{m}\left[\dot{x}^{k} \Gamma_{k h}^{i}-c_{i h} \dot{\widehat{x}}^{k} \widehat{\Gamma}_{k h}^{i}\right]\right)^{2}
$$

where $c_{i h}=\sum_{r, s=1}^{m} \varepsilon_{r} \varepsilon_{s} a_{r h} a_{s i}$. 
Analogously for $\dot{B}(t)=B(t) \cdot \mathcal{U}(t)$, where $B=\left\{b_{\kappa \lambda}\right\}_{\kappa \lambda=1}^{\mathbf{m}}$ is a curve in the group $G_{\nu-\mu}(\mathbf{m})$ and $\mathcal{U}$ is a curve in the Lie algebra of $G_{\nu-\mu}(\mathbf{m})$ we have

$$
\begin{aligned}
-\operatorname{tr} \mathcal{U}^{2} & =-\sum_{\kappa, \chi=1}^{\mathbf{m}}-\varepsilon_{\kappa} \varepsilon_{\chi} w_{\kappa \chi}^{2} \\
& =\sum_{\kappa, \chi=1}^{\mathbf{m}} \varepsilon_{\kappa} \varepsilon_{\chi}\left(\sum_{l=1}^{\mathbf{m}}\left[\dot{x}^{l}\left(\Gamma^{\perp}\right)_{l \chi}^{\kappa}-d_{\kappa \chi} \dot{\widehat{x}}^{l}\left(\widehat{\Gamma}^{\perp}\right)_{l \chi}^{\kappa}\right]\right)^{2},
\end{aligned}
$$

where $d_{\kappa \chi}=\sum_{\rho, \sigma=1}^{\mathbf{m}} \varepsilon_{\rho} \varepsilon_{\sigma} b_{\rho \chi} b_{\sigma \kappa}$.

\section{References}

[1] A. Agrachev and Y. Sachkov, Control Theory from the Geometric Viewpoint, Springer, 2004.

[2] R. Bryant and L. Hsu, Rigidity of integral curves of rank 2 distributions, Invent. Math. 114 (1993), no. 2, 435-461.

[3] Y. Chitour and P. Kokkonen, Rolling of Manifolds and Controllability in dimension three, accepted in Memoires of SMF.

[4] C. J. S. Clarke, On the global isometric embedding of pseudoRiemannian manifolds, Proc. Roy. Soc. Lond. A. 314 (1970), 417-428.

[5] E. Grong, Controllability of rolling without twisting or slipping in higher dimensions, SIAM J. Control Optim. 50 (2012), no. 4, 2462-2485.

[6] M. Godoy Molina and E. Grong, Geometric conditions for the existence of an intrinsic rolling, Commun. Pure Appl. Anal. 13 (2014), no. 1, 435-452.

[7] M. Godoy Molina, E. Grong, I. Markina, and F. Silva Leite, Intrinsic rolling of manifolds, in: Proceedings CONTROLO'2010, 8-10 September 2010, University of Coimbra, Portugal.

[8] M. Godoy Molina, E. Grong, I. Markina, and F. Silva Leite, An intrinsic formulation of the problem on rolling manifolds, Internat. J. Dyn. Control Syst. 18 (2012), no. 2, 181-214.

[9] A. Korolko and F. Silva Leite, Kinematics for rolling a Lorentzian sphere, in: Proc. 50th IEEE CDC-ECC, 12-15 December 2011, OrlandoUSA (2011), 6522-6528. 
[10] K. Hüper and F. Silva Leite, On the geometry of rolling and interpolation curves on $S^{n}, \mathrm{SO}_{n}$, and Grassmann manifolds, J. Dyn. Control Syst. 13 (2007), no. 4, 467-502.

[11] K. Hüper, K. Krakowski, and F. Silva Leite, Rolling maps in a Riemannian framework, in: Textos de Matemática, Volume 43 (J. Cardoso, K. Hüper, P. Saraiva, Eds.), Departamento de Matemática da Universidade de Coimbra, Portugal, 2011, 15-30.

[12] B. O'Neill, Semi-Riemannian geometry, Academic Press, Elsevier 1983.

[13] R. W. Sharpe, Differential Geometry, GTM, 166. Springer-Verlag, New York, 1997.

[14] J. A. Zimmerman, Optimal control of the sphere $S^{n}$ rolling on $E^{n}$, Math. Control Signals Systems 17 (2005), no. 1, 14-37.

Department of Mathematics, University of Bergen, Norway

E-mail address: irina.markina@uib.no

Department of Mathematics and Institute of Systems and Robotics

University of Coimbra, Portugal

E-mail address: fleite@mat.uc.pt

Received May 28, 2014 